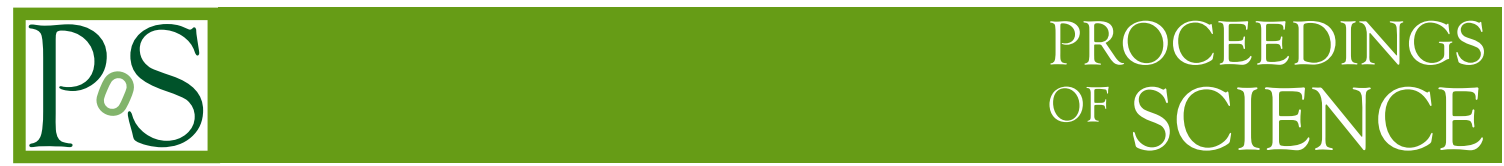

\title{
A charged Z' to explain the apparent disagreement in top-antitop asymmetries between Tevatron and LHC
}

\author{
Estefania Coluccio Leskow* \\ Buenos Aires University \\ E-mail: ecolucciol@gmail.com
}

\section{Ezequiel Álvarez}

Buenos Aires University

E-mail: sequi76@gmail.com

\begin{abstract}
We propose a model with a charged, electrically neutral, flavour changing $Z^{\prime}$ boson to conciliate the apparent disagreement between the excess found in the $t \bar{t}$ forward-backward asymmetry at the Tevatron and the null -compatible with negative- results for the charge asymmetry at the LHC. We show that this model predicts a positive forward-backward asymmetry, while naturally a new cancellation is turned on at the LHC, yielding a null, or even negative, charge asymmetry. We find the region in parameter space that is simultaneously allowed by the most constrictive Tevatron and LHC observables. We show that the model is safe to atomic parity violation constraints and we propose a possible increase in the $Z^{\prime}$ width in order to avoid $t \bar{t} j$ constraints. We finally evaluate the constraints to the model, as well as distinctive features in the forthcoming experimental results.
\end{abstract}

36th International Conference on High Energy Physics

4-11 July 2012

Melbourne, Australia

${ }^{*}$ Speaker. 


\section{Introduction}

The study of the top quark could be crucial to understand the electroweak symmetry breaking mechanism and to discover New Physics (NP). Probably one of the most striking experimental result that can give us hints of NP effects in this sector is the excess measured in the $p \bar{p} \rightarrow t \bar{t}$ forward-backward asymmetry $\left(A_{F B}\right)[1,2]$.

Many NP models [3] arose to account for this excess which, if generated by new physics, could be tested at the LHC where the top quark forward-backward asymmetry vanishes (because it is a symmetric $p p$ collider) and a charge asymmmetry $\left(A_{C}\right)$ is measured. The results found in the LHC charge asymmetry are null -compatible with negative- and compatible with the theory.

In this work we study a phenomenological charged $Z^{\prime}$ model with flavour violating couplings to $u$ and $t$ quarks. We analyse its Tevatron and LHC phenomenology and verify that there is a cancellation of the charge asymmetry, making possible the simultaneous explanation not only of both $A_{F B}$ and $A_{C}$, but also of all CDF unfolded results, APV, and LHC $t \bar{t}$ cross section within the $95 \%$ C.L. The model predicts an excess in $t \bar{t} j$ final state which can be avoided increasing the $Z^{\prime}$ width, assuming the $Z^{\prime}$ decays to not detectable particles a fraction of the times. This new feature of the model predicts single top production with a particular topology.

\section{The model, its phenomenology and results}

The interacting part of the phenomenological NP Lagrangian is given by: $\mathscr{L}_{N P}=f_{R} \bar{u} \gamma^{\mu} P_{R} t Z_{\mu}^{\prime}+$ $f_{R} \bar{t} \gamma^{\mu} P_{R} u Z_{\mu}^{\prime \dagger}$, where $P_{R}=\frac{\left(1+\gamma^{5}\right)}{2}$ and $f_{R}$ is the right-handed coupling ${ }^{1}$. It is relevant to note that we are considering a charged boson, so the production of same sign tops is forbidden.

The Feynman diagrams for $p p, p \bar{p} \rightarrow t \bar{t}(u)$ involving a $Z^{\prime}$ boson in this model are shown in Fig. 1 . We denote by $t_{1}$ the diagram where this particle is exchanged through a $t$-channel and by $s_{1}$ and $s_{2}$ those diagrams with the $Z^{\prime}$ going through an $s$-channel. Since $Z^{\prime} \neq Z^{\prime \dagger}, s_{1}$ and $s_{2}$ have different conjugate diagrams, $\bar{s}_{1}$ and $\bar{s}_{2}$, which at the Tevatron, due to the symmetry in $p \leftrightarrow \bar{p}$, have the same strength as $s_{1}$ and $s_{2}$. At the LHC, on the contrary, $\sigma\left(\bar{s}_{1}, \bar{s}_{2}\right) \ll \sigma\left(s_{1}, s_{2}\right)$.
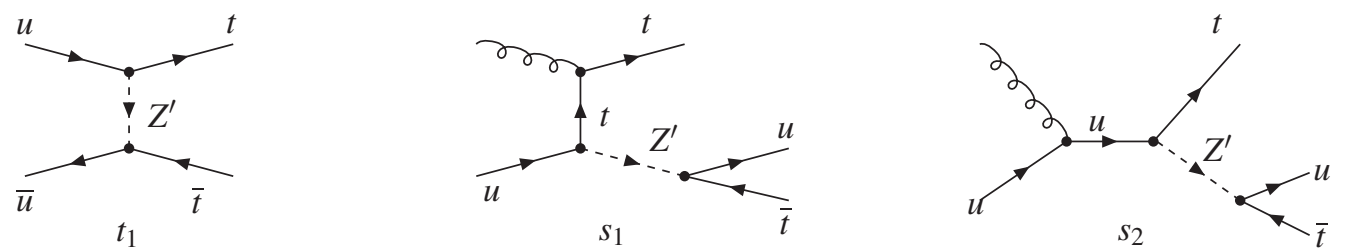

Figure 1: Feynman diagrams for $p p, p \bar{p} \rightarrow t \bar{t}(u)$ involving a $Z^{\prime}$ : In $t_{1}$ the $Z^{\prime}$ is exchanged through a $t$-channel and in $s_{1}$ and $s_{2}$ the $Z^{\prime}$ goes through an $s$-channel. We show that $s_{1}$ cancels $t_{1}$ contribution to the charge asymmetry at the LHC.

The cornerstone of our analysis is the observation that at the LHC a cancellation of the charge asymmetry coming from the positive contribution of the $t$-channel and negative contribution of the $s$-channel processes takes place, explaining the small and compatible with negative charge asymmetry measured by this experiment. This cancellation is not present at the Tevatron where actually a large $A_{F B}$ has been measured.

\footnotetext{
${ }^{1}$ the left-handed coupling is constrained by $B$-physics [4]
} 
We search numerically for this cancellation using MADGRAPH5 [5] and we simulate, in addition to the SM LO $t \bar{t}$ contribution, $t \bar{t}(u)$ production at the Tevatron and the LHC@7TeV within the $Z^{\prime}$ model at parton level according to the diagrams of Fig. 1 and their conjugates. We perform a $\chi^{2}$ test with the last differential measurements of $A_{F B}[6]$ and $\sigma_{t \bar{t}}$ [7] at parton level from CDF, and $\sigma_{t \bar{t}}$ [8] and $A_{C}$ [9] from CMS and confront the model with other relevant constraints in a separate way each.

The last $A_{F B}$ measurement published by $\mathrm{CDF}$ [6] shows $A_{F B}$ as a function of both the invariant mass $M_{t \bar{t}}$ and $\Delta y$. The ranges for the bins used in that analysis, and in our $\chi^{2}$ test, are: $[0-$ $450 ; 450-550 ; 550-650 ; 650-\infty] \mathrm{GeV}$ for $M_{t \bar{t}}$, and $[0-0.5 ; 0.5-1 ; 1-1.5 ; 1.5-\infty]$ for $\Delta y$. We select the points in parameter space which are in agreement with CDF results at a 95\% C.L. by requiring the p-value to be greater than 0.05 . The $M_{Z^{\prime}}$ vs. $f_{R}$ region consistent with Tevatron measurements is delimited by the green dashed lines in all the figures that follow.

With the only purpose of explicitly observing the positive and negative contributions to the charge asymmetry we first analyse each of them separately. ${ }^{2}$.

We show in Fig. 2, the $t$ - (left) and $s$-channel (right) contributions to the charge asymmetry, which we call $A_{C_{t}}$ and $A_{C_{s}}$ respectively. The background colours in the plots indicate the sign of the contribution for every point in the parameter space; red (blue) represents positive (negative) sign. The tone of the colours stands for the absolute value of the contribution; the more intense the tone, the larger the absolute value. The numbers in every point are the difference of $A_{C_{t}}+A^{S M @ N L O} / 2$ (left) and $A_{C_{S}}+A^{S M @ N L O} / 2$ (right) to half the measured value of the charge asymmetry, in units of the experimental error. In the left plot the differences to the measured value are mainly positive while those in the right one are principally negative, what results in the expected cancellation of the charge asymmetry.

We show in Fig. 3 (left) the contributions of both $A_{C_{t}}$ and $A_{C_{s}}$ using the same convention of colours and tones as in the two previous figures with the distinction that now in every cell there are two numbers. The upper one is the difference of $A_{C_{t}}+A_{C_{s}}+A_{C}^{S M @ N L O}$ to the measured value of the charge asymmetry, in units of the experimental error. The number below is the difference of the model prediction for the $t \bar{t}$ inclusive cross-section to the measured value, in units of the error. The triangle delimits the area containing the points consistent with Tevatron limits in which these two observables differ in less than 2 from their corresponding experimental values in units of the experimental error.

\subsection{Constraints and predictions of the model}

This model may come into conflict with APV observables. Ref. [11] studies the limits given by APV in a model with a vector mediator coupled to $u_{R}$ and $t_{R}$ and a flavor-conserving boson. Adapting their constraints to our model, we find the region compatible with APV limits for our model. These limits are given by the thick white line in the left plot of Fig. 3 for a cut-off $\Lambda=$ $1000 \mathrm{GeV}$. In contrast to Ref. [11], in our model the $Z^{\prime}$ mass is larger than the top mass and there is not a light flavor-conserving boson; these two diferences relax the APV constraints. In any case, it is worth to mention that the corrections to the calculation of the parity non-conservation in cesium are currently under discussion [12].

\footnotetext{
${ }^{2}$ For details on the definition of these contributions see [10]
} 

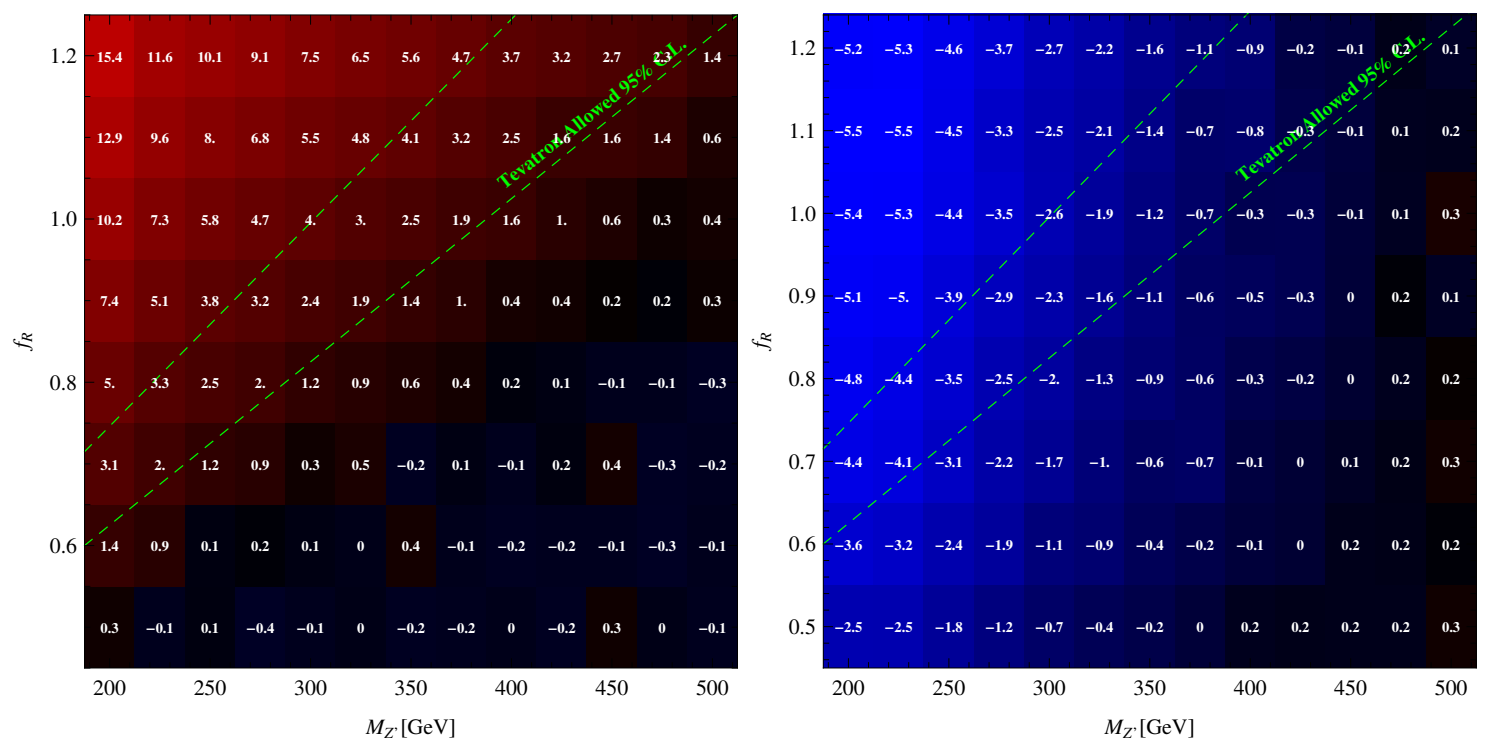

Figure 2: [Color online] (left) $t$-channel contribution to the charge asymmetry and (right) $s$-channel contribution to the charge asymmetry as explained in the text. The dashed green lines delimit the region consistent with Tevatron measurements.
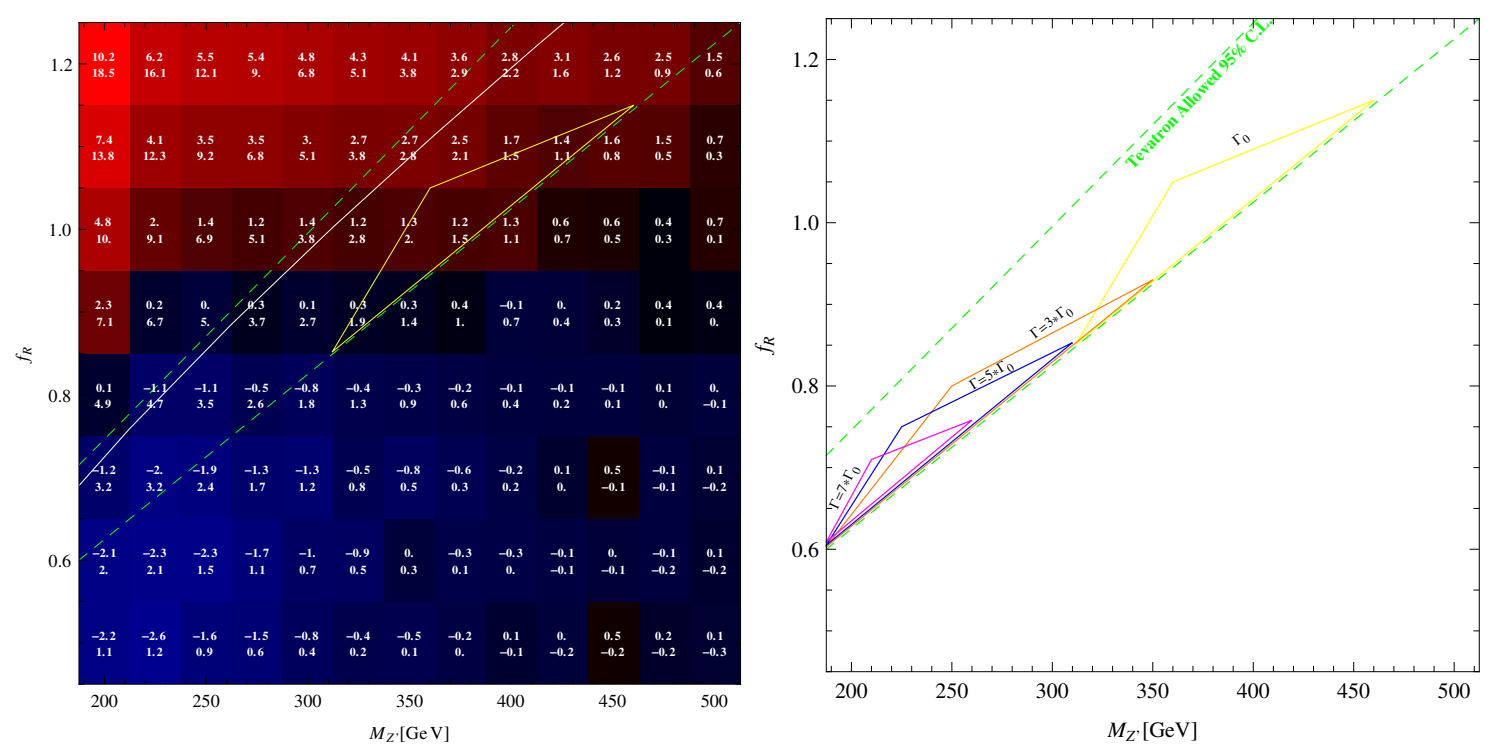

Figure 3: [Color online] (Left) $t$ - and $s$-channel contributions to the charge asymmetry as explained in the text. The parameter space above the thick line shows the APV excluded region. (Right) Regions of the parameter space compatible with all the observables considered in this work from the Tevatron and the LHC for three different increments of the $Z^{\prime}$ width, as explained in the text. We also show the yellow triangle of the left plot that corresponds to no change in the $Z^{\prime}$ width. This increment in the width avoids $t \bar{t} j$ constraints to the model. The dashed green lines delimit the region consistent with Tevatron measurements.

The $t \bar{t} j$ final state brings with it two constraints to the model: one comes from $t \bar{t} j$ production and the second one from $\bar{t} j / t j$ resonance searches. This last constraint is analysed in Ref. [10] but 
not in this work. For $t \bar{t} j$ production, without available works on $t \bar{t} j$ limits that could be adapted to our model, we use $W^{\prime} t d$ production results from Ref. [13] as a rough estimation of the $t \bar{t} j$ production at the LHC in our model. We found that the $Z^{\prime}$ width should be increased to avoid $t \bar{t} j$ constraints. We repeated the simulations for increased values of the $Z^{\prime}$ width and searched again for the allowed parameter space. We show in the right plot of Fig. 3 the allowed region for a $Z^{\prime}$ width three (orange), five (blue) and seven (magenta) times its value when the decay is solely to $u$ and $\bar{t}$, which we denote by $\Gamma_{0}$. We also show in this figure the yellow triangle of the left plot of Fig. 3 that corresponds to no change in the $Z^{\prime}$ width. Note that Tevatron results are not affected by the width modification and that an analysis of the numbers in the left plot of Fig. 3 allows one to conclude that in a given allowed region, in both plots of Fig. 3, $A_{C}$ is the most sensitive observable in the sector of large $M_{Z^{\prime}}$ and $f_{R}$, and $\sigma_{t \bar{t}}$ in the sector of smaller $M_{Z^{\prime}}$ and $f_{R}$. Also notice that the triangles in the right plot of Fig. 3 become smaller with larger values of the $Z^{\prime}$ width suggesting that it cannot be increased arbitrarily because the effect of this increment on either $A_{C}$ or $\sigma_{t \bar{t}}$ (or both) eventually becomes important enough to exclude most of the parameter space [10].

We have checked that increasing the $Z^{\prime}$ width by a factor of 3 avoids the $t \bar{t} j$ constraints of the cited work with $0.7 f b^{-1}$. We have also noted that the $5 f b^{-1}$ projected constraints would not exclude the model if the increment of the width is of a factor of 5. Since $d$ quarks PDFs are different from those of the $u$ quarks, the analysis made in [13] cannot be adapted to our model straightforward and needs a new study.

The increment of the $Z^{\prime}$ width brings with it an excess in single-top production. In order to perform a precise study of this production predicted in our model, one has to look for one reconstructed top, missing energy and no extra $b$ quark [14]. CDF [15] has reported a search of these characteristics, but for masses below those that we find favorable in this work. A full search strategy for the single top topology predicted in this model for the LHC is required [16].

Another specific feature of the model is the dependence of $A_{C}$ with $p_{T}(t \bar{t})$ caused by the different $s$ - and $t$-channel contributions to $A_{C}$ that results in different contributions to the $p_{T}(t \bar{t})$. The $s$-channel processes involve a jet in the final state that provides the $t \bar{t}$ pair with an extra source of $p_{T}(t \bar{t})$ apart from that of the initial state radiation (ISR). This is not the case for the $t$-channel process where the final state is $t \bar{t}$ and no additional sources of $p_{T}$ other than ISR exists. As a result, one expects the $s$-channel to be dominant for large values of $p_{T}(t \bar{t})$. Since the $s$-channel contributes negatively to $A_{C}$, the model then predicts an excessive negative contribution to this observable when events with large $p_{T}(t \bar{t})$ are considered. On the contrary, for low values of $p_{T}(t \bar{t})$ the $t$-channel is preferred and one expects a smaller negative contribution from $A_{C_{s}}$ to the charge asymmetry, i.e., an excess in the positive contributions to $A_{C}$ coming from the $t$-channel processes.

\section{Conclusions}

We have studied a phenomenological model with a new colorless, flavour-violating, electrically neutral $Z^{\prime}$ boson with right handed couplings to $u$ and $t$ quarks. We have shown that this model predicts a large positive $A_{F B}$ as a result of the new particle exchange through a $t$-channel process. Also a null or even negative $A_{C}$ is predicted as a consequence of a negative contribution to this asymmetry by processes turned on at the LHC, in which the new particle goes via an $s$-channel. 
We investigated the allowed parameter space of the model by confronting it with several relevant and most stringent unfolded results from CDF and CMS at $95 \%$ C.L and with its major constraints such as atomic parity violation and $t \bar{t} j$ and single top production. We have shown that our model brings compatibility to the apparent disagreement between $t \bar{t}$ Tevatron forward-backward asymmetry and LHC charge asymmetry.

\section{References}

[1] T. Aaltonen et al. [CDF Collaboration], Evidence for a Mass Dependent Forward-Backward Asymmetry in Top Quark Pair Production, Phys. Rev. D 83, 112003 (2011) [arXiv: 1101.0034 [hep-ex]].

[2] The CDF Collaboration, Conf. Note 10807, http://www-cdf.fnal.gov/physics/new/top/ 2012/LepJet_AFB_Winter2012/CDF10807.pdf; A. Lister [CDF and D0 Collaboration], Top Quark Pair Production Cross Section and Forward-Backward Asymmetry at the Tevatron, arXiv:0810.3350 [hep-ex]; and references therein.

[3] S. Jung, H. Murayama, A. Pierce, J. D. Wells, Top quark forward-backward asymmetry from new t-channel physics, Phys. Rev. D81 (2010) 015004. [arXiv:0907.4112 [hep-ph]];

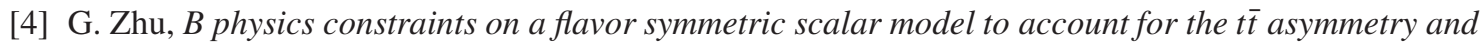
$W_{j j}$ excess at CDF, Phys. Lett. B 703, 142 (2011) [arXiv:1104.3227 [hep-ph] ].

[5] F. Maltoni and T. Stelzer, JHEP 0302, 027 (2003) [arXiv:hep-ph/0208156].

[6] The CDF Collaboration, Conf. Note 10807, http://www-cdf.fnal.gov/physics/new/top/ 2012/LepJet_AFB_Winter2012/CDF10807.pdf

[7] T. Aaltonen et al. [CDF Collaboration], (2009), Public Note 9913.

[8] The CMS Collaboration, CMS-PAS-TOP-11-024.

[9] S. Chatrchyan et al. [CMS Collaboration], Inclusive and differential measurements of the t $\bar{t}$ charge asymmetry in proton-proton collisions at $7 \mathrm{TeV}$, [arXiv:1207.0065 [hep-ex]].

[10] E. Alvarez and E. C. Leskow, A charged Z' to conciliate the apparent disagreement between top-antitop Tevatron forward-backward asymmetry and LHC charge asymmetry, [arXiv:1209.4354 [hep-ph]].

[11] M. I. Gresham, I. -W. Kim, S. Tulin and K. M. Zurek, Confronting Top AFB with Parity Violation Constraints, [arXiv:1203.1320 [hep-ph]].

[12] V. A. Dzuba, J. C. Berengut, V. V. Flambaum and B. Roberts, Revisiting parity non-conservation in cesium, [arXiv:1207.5864 [hep-ph]];

[13] D. Duffty, Z. Sullivan and H. Zhang, Top quark forward-backward asymmetry and W' bosons, Phys. Rev. D 85, 094027 (2012) [arXiv: 1203.4489 [hep-ph] ].

[14] J. Andrea, B. Fuks and F. Maltoni, Monotops at the LHC, Phys. Rev. D 84, 074025 (2011) [arXiv:1106.6199 [hep-ph]].

[15] T. Aaltonen et al. [CDF Collaboration], Search for a dark matter candidate produced in association with a single top quark in p $\bar{p}$ collisions at $\sqrt{s}=1.96 \mathrm{TeV}$, Phys. Rev. Lett. 108, 201802 (2012) [arXiv:1202.5653 [hep-ex]].

[16] E. Alvarez and E. Coluccio Leskow, work in progress. 